\title{
Relative Contribution of Seed-Transmitted Inoculum to Foliar Populations of Phaeosphaeria nodorum
}

\author{
Rebecca S. Bennett, Michael G. Milgroom, Raazesh Sainudiin, Barry M. Cunfer, and Gary C. Bergstrom
}

First, second, and fifth authors: Department of Plant Pathology, Cornell University, Ithaca, NY 14853; third author: Department of Mathematics, Cornell University, Ithaca, NY 14853; and fourth author, Department of Plant Pathology, University of Georgia, Griffin 30223.

Current address of R. S. Bennett: Western Integrated Cropping Systems Research Unit, United States Department of AgricultureAgricultural Research Service, 17053 North Shafter Avenue, Shafter, CA 93263.

Current address of R. Sainudiin: Mathematical Genetics Group, Department of Statistics, University of Oxford, 1 South Parks Road, Oxford, OX1 3TG, United Kingdom.

Accepted for publication 11 December 2006.

\begin{abstract}
Bennett, R. S., Milgroom, M. G., Sainudiin, R., Cunfer, B. M., and Bergstrom, G. C. 2007. Relative contribution of seed-transmitted inoculum to foliar populations of Phaeosphaeria nodorum. Phytopathology 97:584-591.

A marked-isolate, release-recapture experiment was conducted to assess the relative contributions of seed-transmitted (released isolates) versus all other inocula to foliar and grain populations of Phaeosphaeria nodorum in winter wheat rotated with nonsusceptible crops in New York and Georgia, United States. Seed infected with two distinct groups of marked isolates of $P$. nodorum containing rare alleles (identified by amplified fragment length polymorphisms [AFLPs]) and balanced for mating type were planted in experimental field plots in two locations in each state. Recapture was done by isolating $P$. nodorum from leaves showing necrotic lesions at spring tillering and flowering stages, and

sources of the pathogen contributed significant primary inocula to populations recovered from leaves and harvested grain. Seed-transmitted genotypes accounted for a total of $57 \%$ of all isolates recovered from inoculated plots, with a range of 15 to $90 \%$ of the populations of $P$. nodorum collected over the season in individual, inoculated plots at the four locations. Plants in the noninoculated control plots also became diseased and $95 \%$ or more of the isolates recovered from these plots were nonreleased genotypes. Although other potential sources of $P$. nodorum within and adjacent to experimental plots were not ruled out, nonreleased genotypes likely were derived from immigrant ascospores potentially from sources at a considerable distance from the plots. Our results suggest that, although reduction of seedborne inoculum of $P$. nodorum may delay foliar epidemics, this strategy by itself is unlikely to result in high levels of control in eastern North America because of the additional contribution from alternative sources of inoculum.
\end{abstract} mature grains from spikes showing glume blotch. Isolates from these samples were genotyped by AFLPs and categorized as released or nonreleased to infer sources of inoculum. Both infected seed and other
Additional keywords: recombination, Stagonospora nodorum.
Determining sources of primary (or initial) inoculum for epidemics is an integral step toward understanding and managing plant diseases. Primary inoculum may be derived from dormant or survival structures of a pathogen, or from actively growing pathogens from other plant populations. Primary inoculum may preexist in the field or may be introduced once or multiple times; it may continue to arrive and cause primary infections in a plant population even after secondary cycles have begun for polycyclic diseases. Though temporal variation in the arrival of inoculum may complicate the identification of multiple potential sources, identifying the relative contributions of sources of primary inoculum may be crucial for disease management.

In the Phaeosphaeria nodorum (E. Müller) Hedjadroude (anamorph: Stagonospora nodorum (Berk.) Castellani \& E. G. Germano)-wheat pathosystem, the relative contributions of different sources of primary inoculum in rotational cropping systems have not been demonstrated $(42,43)$. Potential sources of primary inoculum include in-field wheat debris, infected gramineous weeds, out-of-field wheat debris, and infected seed. Within-field wheat debris, a significant source of inoculum of $P$. nodorum in

Corresponding author: G. C. Bergstrom; E-mail address: gcb3@ cornell.edu

doi:10.1094/PHYTO-97-5-0584

(C) 2007 The American Phytopathological Society continuous wheat production $(18,20)$, is typically degraded by the time a new wheat crop is planted in most rotational cropping systems. Infected gramineous weeds $(25,45,47)$ are unlikely to be a significant source of inoculum because $P$. nodorum appears to be host-specialized. Isolates of $P$. nodorum derived from wild grasses were found to be significantly less virulent on wheat (25). The two most likely sources of primary inoculum of $P$. nodorum in rotational wheat are infected seed $(2,12,19,29)$ and out-of-field wheat debris generating immigrant airborne ascospores $(1,32,35)$. Once an epidemic of Stagonospora nodorum blotch is initiated by primary inoculum, repeated secondary cycles of asexual or sexual spore production can result in damaging foliar epidemics.

Infected seed may be a major source of primary inoculum for foliar epidemics. Infection of seed by $P$. nodorum is well documented, and seed infection in commercial wheat seed lots is common $(12,19,29)$. In a 1990 sampling of New York commercial seedlots, the mean seed infection incidence was $23 \%$, with a range of 1 to $71 \%$ (37). P. nodorum is transmitted efficiently over a broad range of soil temperatures from infected seed to aerial portions of seedlings where the fungus forms pycnidiospores that are dispersed by splashing rain (39). Plots sown with seed having moderate incidences of infection have resulted in more foliar disease than plots sown from seed that had low or no infection $(3,28,40)$. In addition to showing significant correlations between seed infection and disease severity, Shah et al. (40) tracked geno- 
types of $P$. nodorum from inoculated seed to foliar epidemics, and then to the next generation of wheat seed in the field, providing direct evidence that seed was a source of inoculum.

Immigrant ascospores also may provide primary inoculum for annual epidemics in rotational wheat. Phaeosphaeria ascospores have been trapped in Europe, where Arseniuk et al. (1) trapped them throughout the wheat growth cycle in Poland (collecting most in the fall), and in northern Germany, where ascospores were detected throughout the fall and spring (34). Large quantities of ascospores also were trapped in Australia in July, during active wheat growth (4). Pseudothecia or ascospores of $P$. nodorum also have been reported from Oregon (10), Montana (36), Saskatchewan (15), and North Carolina (11), but their contribution as primary inoculum in these locations has yet to be determined. Sexual structures of $P$. nodorum have not been found in Georgia, Arkansas, or New York despite concerted efforts to look for them. Cunfer (13), using trap plants over 3 years, was unable to find evidence for airborne inoculum in Georgia. Milus and Chalkley (33) were unsuccessful in finding $P$. nodorum ascospores in Burkard traps set out in a wheat field from late November to mid-May in Arkansas. Similarly, in New York, pseudothecia were not found in wheat stubble and ascospores were not found in spore traps set out in a heavily infested field during autumn (L. Litchfield-Kimber and G. C. Bergstrom, unpublished). A possible explanation for the discrepancy among these studies with the results reported from North Carolina (which are all in the eastern U.S. soft winter wheat production region) may be the differential timing of collection efforts. Sampling efforts in New York and Arkansas were focused on autumn through spring, when the lesions and pycnidia typically are first observed on winter wheat, whereas Cowger and Silva-Rojas (11) found the greatest number of pseudothecia on crop debris in the summer months after harvest. Ascospores may serve as primary or secondary inoculum, depending on whether sexual reproduction occurs on debris from a previous crop or on tissues of the current crop. Because crop debris is degraded and essentially absent within wheat fields in rotational systems, ascospores from debris would come from outside a field and be considered as immigrant inoculum in an experimental context, whereas those arising from seedderived isolates in the current crop would be part of the withinfield pool of secondary inoculum. To serve as epidemiologically significant primary inoculum, pseudothecial production or ascospore release may need to occur in autumn or early spring. Later contributions of ascospores from debris during or after crop maturation may have less epidemiological significance because pycnidiospore-driven foliar epidemics would have already progressed to advanced stages under favorable environmental conditions $(14,17,27)$. Regardless of timing, if ascospores constitute a quantitatively large pool of primary inoculum for foliar epidemics, it seems reasonable to expect that sexual structures and ascospores would be abundant and found with some ease in the region either before or during the epidemic.

Foliar lesions caused by $P$. nodorum typically appear in randomly distributed foci in fields in the interval from plant emergence until the beginning of stem elongation (i.e., crop canopy formation) (41). These randomly scattered foci may originate from autumn or spring infections by either seedtransmitted fungus or by airborne spores. Disease development on upper leaves and spikes following stem elongation tends to appear more uniformly within fields (G. C. Bergstrom, unpublished) suggesting secondary inoculum production, expansion, and coalescence of early season foci, or widespread exposure to immigrant, airborne spores at later stages of the epidemic. However, epidemiological observations alone are inadequate to distinguish between airborne and seedborne sources of primary inoculum.

Similarly, population genetic analyses, alone, are inadequate to distinguish between airborne and seedborne, primary inocula in local epidemics. Populations of $P$. nodorum from both wheat leaves and seed have shown high levels of genotypic diversity, gametic equilibrium, equal mating type frequencies, and little significant differentiation across geographic regions $(5,23,24,44)$; these characteristics are consistent with a sexually reproducing fungus with long-distance dispersal of ascospores. However, only small amounts of recombination may be required to maintain the appearance of panmixis $(9,21,31)$ and the lack of geographic differentiation also may be explained by the movement of asexual populations on seed (5). Therefore, although sexual reproduction may affect the pathogen's population genetic structure, it may or may not occur frequently enough or at the right time for ascospores to be a significant source of primary inoculum to initiate annual foliar epidemics. Based on epidemiological and population genetic observations, both seed-transmitted fungus and immigrant ascospores are plausible as primary inocula for foliar epidemics.

Our objective was to determine the relative contribution of seed-transmitted fungus versus all other primary inocula to foliar and grain populations of $P$. nodorum in winter wheat grown in New York and Georgia. By infecting seed with genetically marked isolates, "releasing" them by planting in the field, allowing natural seed-to-seedling transmission, and then "recapturing" them by sampling over the growing season, we estimated the relative contribution of seed-transmitted isolates (i.e., released genotypes) and other isolates (i.e., nonreleased genotypes) as primary inocula in four different wheat environments.

\section{MATERIALS AND METHODS}

Sampling of background populations to find rare-allele isolates. Isolates of $P$. nodorum from New York and Georgia were genotyped to discover rare alleles. A rare allele was defined as an allele with a frequency of $\leq 5 \%$ in each state population. We isolated $P$. nodorum from seed and symptomatic leaves and spikes collected in commercial production fields and university research plots in New York and Georgia from 2000 to 2002. Previous studies have shown little genetic differentiation of the fungus among geographic regions $(5,24)$; therefore, all isolates from various locations in New York and Georgia were considered to represent the $P$. nodorum population for each state. Some of the New York isolates were used in previous studies $(5,6)$. Methods for isolation of $P$. nodorum from infected seed and leaves, culturing conditions, and DNA extraction already have been described $(5,6)$.

We genotyped 472 New York isolates and 119 Georgia isolates for amplified fragment length polymorphisms (AFLP), following a protocol modified for use with fluorescent-labeled primers as previously described $(5,51)$ using the primer pair EcoRI-C/MseICG (5). Data were analyzed with the AFLP analysis option in Genemapper (v. 3.7; Applied Biosystems, Foster City, CA). Chromatograms were checked visually to confirm Genemapper results. Rare alleles were found by screening 80 to 82 unambiguous AFLP loci in the New York and Georgia isolates (5). We screened candidate rare-allele isolates for pathogenicity with a detached leaf assay (26). Only normally pathogenic isolates were used. These isolates also were tested for mating type using MATspecific primers (6).

Production of seed infected with rare-allele isolates. Two arbitrary groups of isolates with unique rare alleles and containing equal numbers of the two mating types then were constructed for both New York and Georgia for a total of four groups: NY-1, NY2, GA-1, and GA-2. In all, 19 rare-allele loci were used in the New York groups and 16 rare-allele loci were used in the Georgia groups. Some isolates had a rare allele at more than one locus. Seed infected with rare-allele isolates were produced in a greenhouse. We sowed seed with the lowest incidence of $P$. nodorum that we could find from winter wheat cvs. Caledonia (soft white) 
and AGS2000 (soft red) for New York and Georgia, respectively. The Caledonia seed had been harvested in a drought year (1999) in New York and treated with $2.3 \mathrm{ml}$ of Raxil-Thiram (tebuconazole $0.6 \%$ and thiram 20\%) per kilogram of seed, a treatment shown to reduce seed transmission of P. nodorum (22). This seed treatment does not result in subsequent protection of wheat leaves from direct infection by $P$. nodorum (G. C. Bergstrom, unpublished). By 2003, we could not detect $P$. nodorum after germinating 1,100 Caledonia seed and screening hypocotyls after 2 weeks. The AGS2000 seed was found to have an infection level by P. nodorum of $0.9 \%$ before seed was treated with Raxil-Thiram. Vernalized plants are necessary for indoor seed production of winter wheat and were generated by the following procedure. Seed were placed on moist blotter paper for 24 to $48 \mathrm{~h}$. Germinated seed were placed in petri dishes with moist filter paper and the dishes then were sealed with parafilm. Seedlings were incubated at $4^{\circ} \mathrm{C}$ for 7 to 8 weeks in the dark. The vernalized seedlings were planted in the greenhouse and were watered only from the bottom of the pots to prevent splash dispersal up the canopy by any $P$. nodorum that may have survived the seed treatment with fungicide. Between anthesis and early milk stage, spikes were partially stripped of their waxy covering by hand and dipped into a $10^{5}$ - to $10^{6}$-conidia $/ \mathrm{ml}$ suspension of $P$. nodorum (plus two drops of Tween 20 per $50 \mathrm{ml}$ of suspension) from each rare-allele isolate. We saved the remaining vernalized plants to generate seed free of $P$. nodorum. After harvest, 30 to 40 seed from inoculated plants were plated on $S$. nodorum agar for wheat (30) to gauge percent infection. We also screened 100 seed from the noninoculated plants.

The greenhouse-produced "clean" seed, and the clean lot of AGS2000 was treated with Raxil-Thiram to further reduce the likelihood of seed-transmitted fungus. We combined the 12 rareallele isolates for each of the four groups in equal amounts so that each isolate could theoretically infect equal numbers of seedlings and produce pycnidiospores on infected foliage. Seed infected with each group of rare-allele isolates was combined with the field-obtained clean lots of Caledonia and AGS2000 to obtain an infection incidence of $10 \%$, a level commonly encountered in commercial seedlots (37). We also harvested sufficient seed from the noninoculated greenhouse plants for use in all control plots. In screening seed from the noninoculated greenhouse plants grown for generating control-plot seed, we did not detect $P$. nodorum in the 100 seeds tested.

Field experiments. The experiment was replicated in two locations in New York (Ithaca and Leroy) and in two locations in Georgia (Griffin and Plains). All locations were in wheat production areas and were $\approx 160 \mathrm{~km}$ apart within states. The plots were planted in September (Ithaca) and October (Leroy) in New York, and in November in Georgia (Griffin and Plains). Three 5by-5-m plots (i.e., inoculated with NY- or GA-1 rare-allele isolates, NY- or GA-2 isolates, or noninoculated, greenhousegenerated control seed) were planted in each location in the middle of a field of nonhost plants to minimize potential inoculum from weedy gramineous hosts. Nonhosts with vertical growth approximating that of wheat were chosen so that they would be similar as barriers for any airborne or splash-dispersed spores. In New York, the plots were planted into first-year alfalfa fields; canola fields were used in Georgia. Plots were separated from each other by $5 \mathrm{~m}$ of nonhost plants; dispersal of pycnidiospores by wind-driven rain has been demonstrated at distances up to $4 \mathrm{~m} \mathrm{(7).} \mathrm{Seed} \mathrm{were} \mathrm{planted} \mathrm{at} \mathrm{a} \mathrm{density} \mathrm{of} 135 \mathrm{~kg} / \mathrm{ha}$ with $18-\mathrm{cm}$ row spacing.

Three samples were taken during the season for $P$. nodorum isolation: a spring foliar sample prior to stem elongation at tillering (Zadoks 30; mid-March in Georgia, late April in New York) (48), a late-spring foliar sample (F-1 or F-2 leaves) around flowering (Zadoks 59-65; early April in Georgia, early June in New York), and a grain sample at harvest (May in Georgia, July in
New York). During the sampling periods, we collected approximately nine diseased leaves or spikes along regular intervals in 25 rows covering the entire area in each plot. In general, only one isolate per leaf or spike was single spored and reserved for genotyping.

Data analyses. We genotyped a subset of recovered isolates to determine their relationship to the released isolates. Overall genetic similarity among recovered isolates to released isolates was examined using 59 polymorphic loci in the New York isolates and 41 polymorphic loci in the Georgia isolates. A recovered isolate was considered as having a released genotype if it had an identical AFLP genotype or if it differed at only one locus. We allowed the margin of error of one locus per genotype to account for scoring error or mutation. Genetic differentiation for each plot over the sampling periods or among the three plots at each location was measured by Weir's $\theta$ (46) with the program MultiLocus (version 1.3b; P.-M. Agapow and A. Burt, Imperial College, London) using 1,000 randomizations.

Because the contribution of seedborne inoculum could be underestimated if released isolates mated with each other, we looked for recombinants that may have originated from mating between released rare-allele isolates using a parental exclusion method. For each recovered isolate with a genotype that differed from those released, we determined whether it could be an offspring of the mating between any possible pair of MAT-1 and MAT-2 released isolates within a plot. A recovered genotype was considered a possible recombinant only if it contained all of the alleles that were common to a pair of MAT-1 and MAT-2 released isolates, and if all other alleles were present in one of the putative parents. This is an unambiguous way of assigning parentage in haploids because we released six isolates of each mating type in each plot and, therefore, had a finite number of matings from which recombinants might occur (8). We also identified possible recombinants with at least one rare allele from one parent as a stricter criterion for recombination between released rare-allele isolates. This criterion results in a systematic underestimation of recombinants because, on average, one-fourth of all recombinants will lack a rare allele (assuming one rare allele at different loci in each parent). Thus, the same analyses were done with all genotypes in background samples from each state to estimate the number of possible recombinants of released isolates we would find by chance in the background populations using these criteria.

\section{RESULTS}

Rare alleles in background populations. We found 19 loci with rare alleles (frequency $<5 \%$ ) among 472 isolates from New York, and 16 loci with rare alleles among 119 isolates from Georgia. Alleles found to be rare in one state were often rare in the other state, but there were also a few rare alleles that were unique to each state (data not shown). Enough isolates of $P$. nodorum carried rare alleles such that we were able to construct two groups of 12 isolates, with 6 of each mating type, for each state.

Climate and disease during the 2003-04 growing season. The two New York locations had average to above-average rainfall over the 2003-04 growing season, resulting in significant foliar epidemics of $P$. nodorum during the grain-filling period. The two Georgia locations had below-average rainfall, resulting in low disease in Plains; supplemental, overhead irrigation was used to produce moderate levels of disease in Griffin. Isolates of $P$. nodorum were not collected from seed in Georgia because incidence of grain infection was very low $(\leq 1 \%$, data not shown). More fungal isolates were recovered from inoculated plots than noninoculated plots at tillering stage using a semiquantitative method of sampling blighted foliage; that trend was not consistent at later sampling periods. Quantitative disease ratings were not made during the growing season for any plots; however, in New York, noninoculated plots were distinguishable from the inocu- 
lated plots at harvest because they had considerably fewer diseased leaves and glumes with symptoms and signs of $P$. nodorum than the inoculated plots.

Recovery of isolates with released and nonreleased genotypes. To directly measure the contribution of infected seed, we looked for recovered isolates with AFLP genotypes identical (or nearly so) to released, rare-allele isolates (Table 1). Genotypes differing from a released genotype by one allele (i.e., nearly identical to released isolates) accounted for 19 and $9 \%$ of all the released isolates recovered in New York and Georgia, respectively. In the background populations, 0 and $2 \%$ of the New York and Georgia isolates, respectively, had genotypes nearly identical to released isolates. Released genotypes were recovered from diseased plants in every inoculated plot at each sampling period (Table 1). Isolates with genotypes identical to those inoculated on seed generally were recovered only from plots where they were released. Over all times and locations, only 23 (6\%) of the 389 isolates recovered with released-isolate genotypes were found from plots in which they were not released. Seed-transmitted genotypes accounted for a total of $57 \%$ of the recovered isolates, with a range of $15 \%$ (Leroy NY-G2) to $90 \%$ (Georgia GA-G2). Conversely, isolates not originating from released genotypes accounted for the remaining isolates recovered from inoculated plots at each location (17 to $69 \%$ ). In the noninoculated control plots, nonreleased genotypes accounted for 90 to $100 \%$ of the foliar isolates recovered (Table 1). Rare alleles in the nonreleased isolates were at background frequencies (data not shown).

In six of the eight inoculated plots, the proportion of isolates with released genotypes did not change significantly over the different sampling periods $\left(\chi^{2}, P \geq 0.10\right)$ (Table 1$)$. The Leroy NY-G2 and Plains GA-G1 plots were exceptions; the proportion of isolates with released genotypes declined over the sampling periods in the Leroy NY-2 plot $\left(\chi^{2}=18.89, P \leq 0.001, \mathrm{df}=2\right)$ and increased in the Plains GA-G1 plot $\left(\chi^{2}=13.25, P \leq 0.001\right.$, df $\left.=1\right)$.

Isolates with nonreleased genotypes were recovered consistently over the sampling periods, and most plots and times had a high diversity of genotypes (Table 1). The Ithaca NY-G1 plot was an exception in that a small number of genotypes constituted a majority of the nonreleased genotypes in the first sampling period. Although a few nonreleased genotypes were collected over multiple sampling periods, most genotypes collected from late foliar and grain samples were novel. We found little evidence of genetic differentiation over the three sampling periods within plots, with or without including isolates with released genotypes (Table 2). A significant difference was found only in the Ithaca NY-G2 plot when released genotypes were included.

Using parental exclusion, 31\% (120/388) and 40\% (44/111) of the nonreleased isolates recovered in New York and Georgia, respectively, were possible recombinants between at least one pair of MAT-compatible released isolates (data not shown). Using the stricter criterion that a recombinant carries at least one rare allele, the frequency of possible recombinants was sharply lower. Only $4 \%(14 / 388)$ and $8 \%(9 / 111)$ of the nonreleased isolates recovered in New York and Georgia, respectively, could have been recombinants by this criterion (data not shown). For comparison, the frequencies of possible recombinants in the original New York and Georgia background populations were not different from those observed in experimental plots by either criteria. In the New York and Georgia background populations, 35\% (159/449) and $52 \%$ (50/95), respectively, were possible recombinants. Frequencies comparable to the background populations also were found among the recovered isolates for the strict definition of recombination: $4 \%$ (20/449) and 4\% (4/95) for New York and Georgia, respectively.

Genetic differentiation among plots for rare allele loci. We found highly significant levels of genetic differentiation among the three plots for each time period for most locations and collection times when released genotypes were included. Only the samples taken from grain in Leroy and from the early foliar samples in Plains were not significantly differentiated (Table 2). When released genotypes were excluded from the analyses, only the Ithaca plots from the early foliar sampling period were significantly different from each other. This is attributable to recovery of 20 isolates of one, nonreleased genotype in the Ithaca NY-1 plot. Lack of significant differentiation for the other locations might be caused, in part, by analysis of smaller sample sizes after excluding released genotypes.

\section{DISCUSSION}

We assessed experimentally the relative contribution of seedtransmitted $P$. nodorum as primary inoculum for foliar epidemics in four different environments by releasing identifiable, genetically marked isolates in infected seed and using greenhouse-generated clean seed for control field plots. Our results demonstrate that both seed-transmitted and other sources of $P$. nodorum contribute significant amounts of primary inocula to foliar populations and subsequent grain populations of the fungus in winter wheat grown in rotation with nonsusceptible crops in the eastern United States. Several results substantiate the strong contribution of seed-transmitted fungus to foliar populations. Seed-transmitted, released genotypes accounted for a range of 15 to $90 \%$ of the populations of $P$. nodorum collected over the season in inoculated plots at the four locations. Though our foliage sampling scheme was not quantitative, plots sown in three of the four locations with infected seed yielded markedly greater numbers of $P$. nodorum isolates in early spring than the control plots that had been planted with the greenhouse-generated clean seed; we interpret this observation to indicate the effect of seedborne inoculum causing more lesions from which to isolate. Although disease severity was not assessed, we observed that the noninoculated control plots in New York continued to show discernibly less necrotic foliage and glumes through crop maturation than in inoculated plots. However, the most compelling evidence for the impact of infected seed is that many recovered isolates were genetically identical to genotypes that had been released in the same plots. Several of these released genotypes were collected multiple times over the growing season (data not shown), confirming that $P$. nodorum isolates in infected seed can establish themselves early, persist through secondary cycles over the season, and eventually infect the next generation of seed, as found previously by Shah et al. (40). More released genotypes were collected from the inoculated plots in Georgia than the plots in New York. Also, there was considerable variation in the percentage of released isolates recovered among inoculated plots within each of the New York locations. Environmental differences (e.g., drought) may have favored seed inoculum in Georgia, and variable fitness of the individual released isolates may have contributed to variation among plots within a location.

The large percentage of isolates recovered from inoculated plots that had nonreleased genotypes is evidence that an immigrant source of primary inoculum contributed significantly to epidemics. Further compelling evidence for primary inoculum that is not seed-transmitted came from the noninoculated control plots in all four locations. Plants in control plots still became diseased despite having originated from seed generated in the greenhouse in the absence of detectable inoculum levels. Nearly all of the multilocus genotypes of isolates recovered from the noninoculated plots were different from those of released isolates; only $3 \%$ of the isolates recovered from noninoculated plots had releasedisolate genotypes (possibly from pycnidiospores dispersed by wind-driven rain, as demonstrated by Brennan et al. [7] in a rain tower-wind tunnel apparatus at distances up to $4 \mathrm{~m}$ ). Rare alleles, if present in the nonreleased isolates, were at frequencies comparable to those in the background populations. Additionally, there was no significant genetic differentiation among nonreleased genotypes from the different plots in 9 of 10 comparisons, indicating a diverse 
TABLE 1. Analyses of amplified fragment length polymorphism (AFLP) genotypes of isolates of Phaeosphaeria nodorum recovered from infected leaves and grains during the growth of winter wheat in New York and Georgia

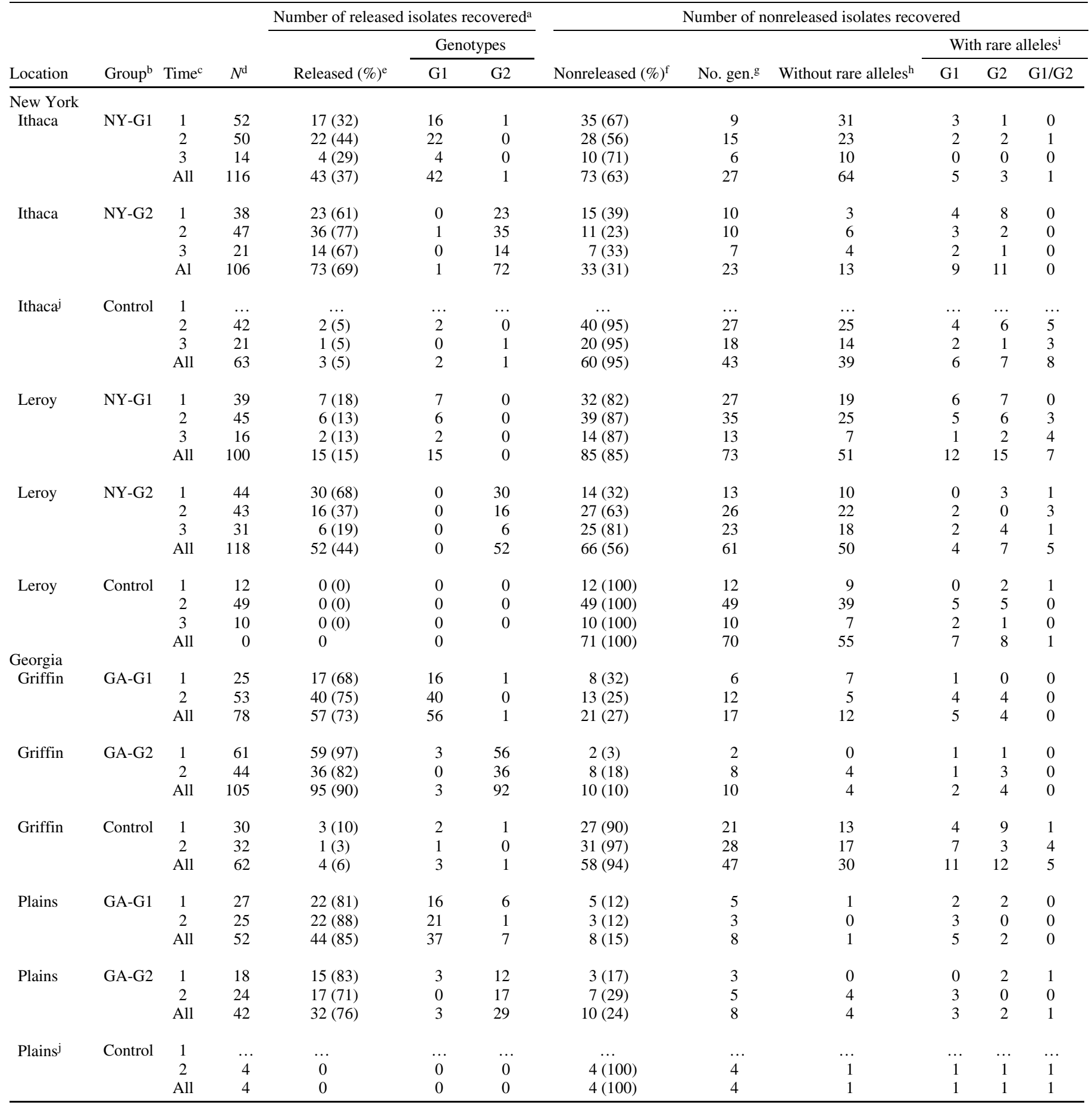

${ }^{a}$ Number of isolates with genotypes identical (or nearly identical, i.e., different at one AFLP locus) to a released isolate genotype. G1 and G2 indicate released genotypes from Group 1 or Group 2.

${ }^{\mathrm{b}}$ Rare allele group. Four groups of rare-allele isolates were released after being inoculated on clean wheat seed. Two groups of isolates with specific AFLP genotypes were released in each state. Isolates collected from the genotypes are referred to as NY-G1 and NY-G2 for New York, and as GA-G1 and GA-G2 for Georgia. Control plots were planted with greenhouse-produced "clean" seed that was not inoculated and in which P. nodorum was not detected.

${ }^{\mathrm{c}}$ Sampling time $=1$, early spring, foliar sampling during tillering; 2 , late spring, foliar sampling during flowering; 3 , sampling of mature grains; and all, data combined for all sampling times.

${ }^{\mathrm{d}}$ Number of isolates genotyped.

e All released isolates $(\%$ of $N$ ).

f All nonreleased isolates $(\%$ of $N)$.

g Number of nonreleased (clone-corrected) genotypes.

${ }^{\mathrm{h}}$ Number of isolates without any rare alleles found in released isolates.

${ }^{i}$ Number of isolates with rare alleles from released genotypes in group 1 (G1), group 2 (G2), or both groups (G1/G2).

j Only four isolates were collected from these plots so they were not genotyped. 
background population contributing inoculum equally to all plots within most locations. In the inoculated plots, the proportion of nonreleased to released genotypes did not change significantly over time, suggesting that population structure was established early and that there was not a large influx of new genotypes late in the epidemic. There was an increased recovery of $P$. nodorum isolates from noninoculated plots in the sampling period between spring tillering and flowering. This may be attributed to secondary cycles of within-plot inoculum increase or to a late-spring influx of primary inoculum, possibly in the form of windborne ascospores. However, hundreds of isolates would need to be sampled at each sampling time to have enough statistical power to detect all but very large changes in the proportions of released and nonreleased genotypes. Therefore, we cannot determine when immigrant, primary inoculum arrived in the plots or whether its con- tribution to the foliar and grain populations increased over time. Aerial spore trapping above the rain-splash dispersal height of plots may be a more direct method for addressing questions of the timing of ingress of airborne primary inoculum.

The origin of nonreleased isolates was not determined; however, there are several potential sources: (i) preexisting, withinplot genotypes of $P$. nodorum that evaded our attempts to generate clean seed or to eliminate grass hosts or their debris; (ii) ascospores arising from sexual recombination of our released isolates (recall that equal numbers of isolates of both mating types were released); and (iii) wind-dispersed ascospores or other fungal propagules originating from outside our plots. Clean commercial seed used in this study was shown to have less than 1.0 and $0.1 \%$ infection incidence from Georgia and New York, respectively. However unlikely, we cannot rule out clean seed as a source of

TABLE 2. Genetic differentiation of recovered isolates at rare-allele amplified fragment length polymorphism (AFLP) loci ${ }^{\mathrm{a}}$

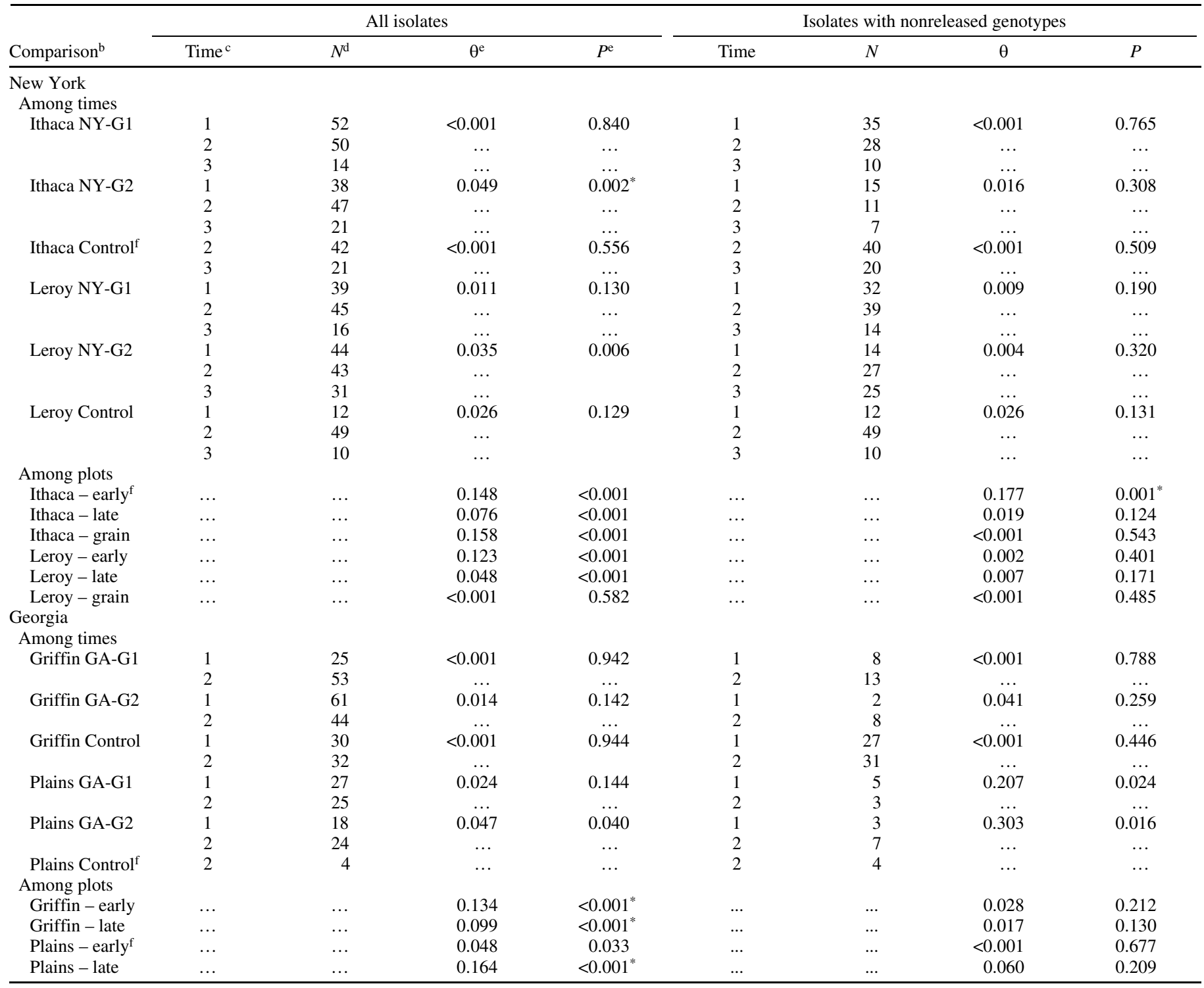

${ }^{a}$ Differentiation was estimated on two sets of data: AFLP allele frequencies based on all of the recovered isolates (including isolates with released genotypes) and on recovered isolates without released genotypes.

${ }^{b}$ Groups of isolates with specific AFLP genotypes were released in each state; Their genotypes are referred to as NY-G1 and NY-G2 for New York, and GA-G1 and GA-G2 for Georgia. Control plots were planted with greenhouse-produced "clean" seed that was not inoculated and no P. nodorum could be detected. Two types of comparisons were made: (i) differentiation among sampling times within plots (Among times), and (ii) differentiation among treated plots at the same time (Among plots). All tests were performed on differentiation within each location.

c Sampling time = 1, early foliar sampling during tillering; 2, late foliar sampling during flowering; and 3, grain at harvest.

${ }^{\mathrm{d}}$ Number of isolates genotyped.

e Weir's estimate of $F_{\mathrm{ST}}$ for haploids (46); $P$ values estimated with 1,000 randomizations (MultiLocus version 1.3b, P.-M. Agapow and A. Burt, Imperial College, London); ${ }^{*}$ indicates significance at $P \leq 0.0023$ (with Bonferroni correction for 22 comparisons for $\alpha=0.05$ ).

${ }^{f}$ No data from control plots for these locations at early sampling period because only three to four isolates were collected. 
nonreleased genotypes into both inoculated and control plots, albeit at a low frequency. Wild grasses and grass debris occur commonly in agricultural settings; therefore, we cannot completely rule out that they provided some small fraction of inoculum from within or near the plots. Although some of the nonreleased genotypes were potential recombinants of mating typecompatible pairs of released isolates, their frequencies were not different from those found for the background population of $S$. nodorum in each state. Therefore, these potential recombinant genotypes cannot be interpreted as evidence for recombination among released isolates. Furthermore, if recombinants were generated among isolates released on infected seed and contributed to secondary cycles of foliar epidemics, they would have been derived ultimately from primary inoculum in infected seed and not from immigrant ascospores. Therefore, recombination among released isolates is irrelevant for identifying the sources of primary inoculum. In summary, we can fairly confidently rule out within-field ascospores (from seedborne isolates), contaminated seed, and within-field debris or grass hosts as significant sources of primary inoculum.

The remaining — and most likely_-source of nonreleased genotypes was immigrant, windborne ascospores. Others $(16,23)$ have proposed ascospores as the principal form of primary inoculum. However, our results show unambiguously that windborne ascospores, although likely significant, are not the sole source of primary inoculum when infected seed is present. A future challenge will be to acquire physical evidence of ascospores through spore trapping above wheat canopies and to determine the timing of their deposition between wheat emergence and stem elongation. The fact that population structure was already established and nonreleased isolates were present by the time of tillering in early spring in our study suggests that immigrant ascospores should be detected in autumn prior to crop dormancy or in early spring before stem elongation. Our previous attempts have failed to find evidence of ascocarps or ascospores of $P$. nodorum in Georgia (13) and New York (L. Litchfield-Kimber and G. C. Bergstrom, unpublished). Recently, Cowger and Silva-Rojas (11) reported finding ascocarps of $P$. nodorum, albeit at low frequentcies, in North Carolina. However, the greatest numbers of pseudothecia, in samples from early summer to mid-fall, were found in late July, long before the next winter wheat crop was planted.

The key to reducing the economic impact of $P$. nodorum on wheat yield is to employ management practices that reduce disease on the upper leaves during grain formation. We found that isolates of $P$. nodorum sampled from the upper leaves and mature seed were derived from seed-transmitted, primary inoculum as well as from other primary inocula. Shah and Bergstrom (38) suggested that a seed health management approach combining clean seed, fungicidal seed treatment, and genetic resistance to seed transmission might result in significant disease control in the absence of substantial immigrant inoculum of $P$. nodorum. The current results suggest that, because immigrant isolates are also important as primary inoculum, seed health strategies alone will be insufficient for management of foliar epidemics. Wheat cultivar resistance to $P$. nodorum or the use of efficacious foliar fungicides, in combination with seed health strategies, likely will be needed for season-long management of Stagonospora nodorum blotch.

The marked isolate, release-recapture experimental approach provided valuable insights into the relative importance of seedtransmitted and other primary inocula in foliar epidemics of $P$. nodorum. The appropriate design of release-recapture experiments and the choice of marked isolates has been debated previously $(8,49,50)$. Our experiment utilized multiple isolates $(24$ total per state) of both mating types and rare-allele loci (19 and 16 in New York and Georgia, respectively). Even with this large number of isolates, some subsets of isolates (i.e., GA-G2 and NY$\mathrm{G} 2$ ), despite prescreening for pathogenicity, appeared to have a higher overall fitness than the others, and some genotypes were not recaptured at all. In agricultural systems, there is even greater variability in the fungus than we represented in our sets of marked isolates. It seems, therefore, that utilization of a large number of arbitrarily chosen marked isolates is necessary for this experimental approach. The combined approaches of population genetic analysis and field epidemiology should find wider application in answering questions about primary inoculum sources in other fungal diseases of plants.

\section{ACKNOWLEDGMENTS}

This work was supported by the United States Department of Agriculture NRICGP grant 2002-35319-12689. We thank S. Kresovich's staff at the Cornell Institute for Genomic Diversity for technical support; J. Youmans at the University of Georgia, Griffin campus, for field support; S. Kawamoto, Q. Arntsen, and M. Sorrell's staff at Cornell University for greenhouse and laboratory support; and anonymous reviewers for helpful feedback.

\section{LITERATURE CITED}

1. Arseniuk, E., Góral, T., and Scharen, A. L. 1998. Seasonal patterns of spore dispersal of Phaeosphaeria spp. and Stagonospora spp. Plant Dis. 82:187-194.

2. Babadoost, M., and Hebert, T. T. 1984. Factors affecting infection of wheat Triticum aestivum seedlings by Septoria nodorum. Phytopathology 74:592-595.

3. Babadoost, M., and Hebert, T. T. 1984. Incidence of Septoria nodorum in wheat seed and its effects on plant growth and grain yield. Plant Dis. 68:125-129.

4. Bathgate, J. A., and Loughman, R. 2001. Ascospores are a source of inoculum of Phaeosphaeria nodorum, $P$. avenaria $\mathrm{f}$. sp avenaria and Mycosphaerella graminicola in Western Australia. Australas. Plant Pathol. 30:317-322.

5. Bennett, R. S., Milgroom, M. G., and Bergstrom, G. C. 2005. Population structure of seedborne Phaeosphaeria nodorum on New York wheat. Phytopathology 95:300-305

6. Bennett, R. S., Yun, S.-H., Lee, T. Y., Turgeon, B. G., Arseniuk, E., Cunfer, B. M., and Bergstrom, G. C. 2003. Identity and conservation of mating type genes in geographically diverse isolates of Phaeosphaeria nodorum. Fungal Genet. Biol. 40:25-37.

7. Brennan, R. M., Fitt, B. D. L., Taylor, G. S., and Calhoun, J. 1985. Dispersal of Septoria nodorum pycnidiospora by simulated rain and wind. J. Phytopathol. 112:291-297.

8. Brown, J. K. M. 2000. Estimation of rates of recombination and migration in populations of plant pathogens. Phytopathology 90:320-323.

9. Burt, A., Carter, D. A., Koenig, G. L., White, T. J., and Taylor, J. W. 1996. Molecular markers reveal cryptic sex in the human pathogen Coccidioides immitis. Proc. Natl. Acad. Sci. USA 93:770-773.

10. Cowger, C., McDonald, B. A., and Mundt, C. C. 2002. Frequency of sexual reproduction by Mycosphaerella graminicola on partially resistant wheat cultivars. Phytopathology 92:1175-1181.

11. Cowger, C., and Silva-Rojas, H. V. 2006. Frequency of Phaeosphaeria nodorum, the sexual stage of Stagonospora nodorum, on winter wheat in North Carolina. Phytopathology 96:860-866.

12. Cunfer, B. M. 1978. The incidence of Septoria nodorum in wheat seed. Phytopathology 68:832-835.

13. Cunfer, B. M. 1998. Seasonal availability of inoculum of Stagonospora nodorum in the field in the Southeastern U.S. Cereal Res. Commun. 26:259-263.

14. Djurle, A., Ekbom, B., and Yuen, J. E. 1996. The relationship of leaf wetness duration and disease progress of glume blotch, caused by Stagonospora nodorum, in winter wheat to standard weather data. Eur. J. Plant Pathol. 102:9-20.

15. Duczek, L. J., Sutherland, K. A., Reed, S. L., Bailey, K. L., and Lafond, G. P. 1999. Survival of leaf spot pathogens on crop residues of wheat and barley in Saskatchewan. Can. J. Plant Pathol. 21:165-173.

16. Eyal, Z. 1999. The Septoria tritici and Stagonospora nodorum blotch diseases of wheat. Eur. J. Plant Pathol. 105:629-641.

17. Gilbert, J., Woods, S. M., and Tekauz, A. 1998. Relationship between environmental variables and the prevalence and isolation frequency of leaf-spotting pathogens in spring wheat. Can. J. Plant Pathol. 20:158-164.

18. Harrower, K. M. 1974. Survival and regeneration of Leptosphaeria nodorum in wheat debris. Trans. Br. Mycol. Soc. 63:527-533.

19. Hewett, P. D. 1965. A survey of seed-borne fungi of wheat .I. Incidence of Leptosphaeria nodorum and Griphosphaeria nivalis. Trans. Br. Mycol. 
Soc. 48:59-72.

20. Holmes, S. J. I., and Colhoun, J. 1975. Straw-borne inoculum of Septoria nodorum and $S$. tritici in relation to incidence of disease on wheat plants. Plant Pathol. 24:63-66.

21. Hudson, R. R. 1994. Analytical results concerning linkage disequilibrium in models with genetic transformation and conjugation. J. Evol. Biol. 7:535-548.

22. Kawamoto, S. O., Bergstrom, G. C., Cox, W. J., and Otis, D. J. 2000. Effects of chemical and biological seed treatments on seed-transmitted Stagonospora nodorum blotch and agronomic performance of winter wheat. Fungic. Nematicide Tests 55:472.

23. Keller, S. M., McDermott, J. M., Pettway, R. E., Wolfe, M. S., and McDonald, B. A. 1997. Gene flow and sexual reproduction in wheat glume blotch pathogen Phaeosphaeria nodorum (anamorph Stagonospora nodorum). Phytopathology 87:353-358.

24. Keller, S. M., Wolfe, M. S., McDermott, J. M., and McDonald, B. A. 1997. High genetic similarity among populations of Phaeosphaeria nodorum across wheat cultivars and regions of Switzerland. Phytopathology 87:1134-1139.

25. Krupinsky, J. M. 1997. Aggressiveness of Stagonospora nodorum isolates from perennial grasses on wheat. Plant Dis. 81:1032-1036.

26. Krupinsky, J. M. 1997. Aggressiveness of Stagonospora nodorum isolates obtained from wheat in the northern Great Plains. Plant Dis. 81:1027-1031.

27. Leath, S., Scharen, A. L., Lund, R. E., and Dietz-Holmes, M. E. 1993. Factors associated with global occurrences of Septoria nodorum blotch and Septoria tritici blotch of wheat. Plant Dis. 77:1266-1270.

28. Luke, H. H., Barnett, R. D., and Pfahler, P. L. 1985. Influence of soil infestation, seed infection, and seed treatment on Septoria nodorum blotch of wheat. Plant Dis. 69:74-76.

29. Machacek, J. E., and Wallace, H. A. H. 1952. Longevity of some common fungi in cereal seed. Can. J. Bot. 30:164-169.

30. Manandhar, J. B., and Cunfer, B. M. 1991. An improved selective medium for the assay of Septoria nodorum from wheat seed. Phytopathology 81:771-773.

31. Maynard Smith, J. 1994. Estimating the minimum rate of genetic transformation in bacteria. J. Evol. Biol. 7:525-534.

32. Mehta, Y. R. 1975. Leptosphaeria nodorum on wheat in Brazil and its importance. Plant Dis. Rep. 59:404-406.

33. Milus, E. A., and Chalkley, D. B. 1997. Effect of previous crop, seedborne inoculum, and fungicides on development of Stagonospora blotch. Plant Dis. 81:1279-1283.

34. Mittelstädt, A., and Fehrmann, H. 1987. The occurrence of the perfect stage of Septoria nodorum in the Federal Republic of Germany. Z. Pflanzenkrankh. Pflanzenschutz 94:380-385.

35. Sanderson, F. R., and Hampton, J. G. 1978. Role of perfect states in epidemiology of common Septoria diseases of wheat. N. Z. J. Agric. Res. 21:277-281.

36. Scharen, A. L., and Sanderson, F. R. 1982. Leptosphaeria nodorum and
Mycosphaerella graminicola in North America. Phytopathology 72:934934.

37. Shah, D. A., and Bergstrom, G. C. 1993. Assessment of seed-borne Stagonospora nodorum in New York soft white winter wheat. Plant Dis. 77:468-471.

38. Shah, D. A., and Bergstrom, G. C. 1999. Epidemiology of seedborne Stagonospora nodorum: A case study on New York winter wheat. Pages 102-104 in: Septoria and Stagonospora Diseases of Cereals: A Compilation of Global Research. M. van Ginkel, A. McNab, and J. Krupinsky, eds. CIMMYT, Mexico, D.F.

39. Shah, D. A., and Bergstrom, G. C. 2000. Temperature dependent seed transmission of Stagonospora nodorum in wheat. Eur. J. Plant Pathol. 106:837-842.

40. Shah, D. A., Bergstrom, G. C., and Ueng, P. P. 1995. Initiation of Septoria nodorum blotch epidemics in winter wheat by seedborne Stagonospora nodorum. Phytopathology 85:452-457.

41. Shah, D. A., Bergstrom, G. C., and Ueng, P. P. 2001. Foci of Stagonospora nodorum blotch in winter wheat before canopy development. Phytopathology 91:642-647.

42. Shaner, G., and Buechley, G. 1995. Epidemiology of leaf blotch of soft red winter wheat caused by Septoria tritici and Stagonospora nodorum. Plant Dis. 79:928-938.

43. Shaw, M. W. 1999. Epidemiology of Mycosphaerella graminicola and Phaeosphaeria nodorum: An overview. Pages 93-97 in: Septoria and Stagonospora Diseases of Cereals: A Compilation of Global Research. M. van Ginkel, A. McNab, and J. Krupinsky, eds. CIMMYT, Mexico.

44. Sommerhalder, R. J., McDonald, B. A., and Zhan, J. 2006. The frequencies and spatial distribution of mating types in Stagonospora nodorum are consistent with recurring sexual reproduction. Phytopathology 96:234-239.

45. Weber, G. F. 1922. Septoria diseases of cereals. II. Septoria diseases of wheat. Phytopathology 12:537-585.

46. Weir, B. S. 1996. Genetic Data Analysis II. Sinauer Assoc. Inc., Sunderland, MA.

47. Williams, J. R., and Jones, D. G. 1973. Infection of grasses by Septoria nodorum and S. tritici. Trans. Br. Mycol. Soc. 60:355-358.

48. Zadoks, J. C., Chang, T. T., and Konzak, C. F. 1974. A decimal code for the growth stages of cereals. Weed Res. 14:415-421.

49. Zhan, J., Mundt, C. C., and McDonald, B. A. 1998. Measuring immigration and sexual reproduction in field populations of Mycosphaerella graminicola. Phytopathology 88:1330-1337.

50. Zhan, J., Mundt, C. C., and McDonald, B. A. 2000. Estimation of rates of recombination and migration in populations of plant pathogens-a reply. Phytopathology 90:324-326.

51. Zhao, S. H., Mitchell, S. E., Meng, J. H., Kresovich, S., Doyle, M. P., Dean, R. E., Casa, A. M., and Weller, J. W. 2000. Genomic typing of Escherichia coli O157: H7 by semi-automated fluorescent AFLP analysis. Microbes Infect. 2:107-113. 